\title{
A COMUNICAC̣ÃO DE MÁS NOTÍCIAS EM SAÚDE: APROXIMAÇÕES COM A ABORDAGEM CENTRADA NA PESSOA
}

\author{
The communication of bad news in health: approximations with the person-centered approach
}

La comunicación de malas noticias en salud: aproximaciones con el enfoque centrado en la persona

VANESSA JaQUeline CALSAVARA FABIO SCORSOLINI-COMIN Carlos Alexandre Curylofo Corsi

\begin{abstract}
Resumo: A forma de comunicar más notícias impacta diretamente na adesão ao tratamento por parte do paciente. Frente às dificuldades em comunicar más notícias, foram elaborados protocolos que auxiliassem os profissionais de saúde nesses momentos de maior vulnerabilidade. Este estudo teórico teve por objetivo aproximar as atitudes/ condições facilitadoras descritas originalmente por Carl Rogers no contexto psicoterápico a três protocolos de comunicação de más notícias frequentemente relatados na literatura da área: SPIKES, CLASS e P-A-CI-E-N-T-E. Diversas etapas desses protocolos emergem como ações que se aproximam ora da congruência/ autenticidade, ora da consideração positiva incondicional ou da empatia, tornando lícito afirmar que a comunicação de más notícias também possibilita o estabelecimento de uma relação de ajuda. Conclui-se que esta associação entre os protocolos e as três atitudes elaboradas por Rogers pode contribuir para a formação acadêmica dos profissionais de saúde e também no estabelecimento de um melhor vínculo entre médico, paciente e familiares. Palavras-chave: Comunicação em saúde; Relações médico-paciente; Terapia centrada na pessoa.
\end{abstract}

Abstract: The way of communicating bad news directly impacts in the treatment adherence of the patient. In light of the difficulties of communicating bad news, protocols were developed to assist the health care professionals in these moments of major vulnerability. This theoretical study aimed to approach the core conditions/attitudes described originally by Carl Rogers in the psychotherapic context to three bad news communication protocols reported from the field specific literature: SPIKES, CLASS and P-A-C-I-E-N-T-E. Diverse steps of these protocols emerge as actions that approximate one moment to congruency/authenticity and another moment to unconditional positive consideration or empathy. It makes licit to affirm that the communication of bad news also approaches to the establishment of a help relation. It can be concluded that this approach between the protocols and the three attitudes developed by Rogers can contribute to the academic training of the health care professionals and also to the better connection between doctor, patient and family. Keywords: Health communication; Physician-patient relations; Person-centered therapy.

Resumen: La forma de comunicar malas noticias impacta directamente en la adhesión al tratamiento por parte del paciente. Frente a las dificultades en comunicar malas noticias, se elaboraron protocolos que auxiliar a los profesionales de salud en esos momentos de mayor vulnerabilidad. Este estudio teórico tuvo por objetivo aproximar las actitudes / condiciones facilitadoras descritas originalmente por Carl Rogers en el contexto psicoterápico con tres protocolos de comunicación de malas noticias a menudo relatados en la literatura del área: SPIKES, CLASS y P-A-C-I-E-N-T-E. Diversas etapas de esos protocolos emergen como acciones que se aproximan a la congruencia / autenticidad, ya la consideración positiva incondicional o de la empatía, haciendo lícito afirmar que la comunicación de malas noticias también posibilita el establecimiento de una relación de ayuda. Se concluye que esta asociación entre los protocolos y las tres actitudes elaboradas por Rogers puede contribuir a la formación académica de los profesionales de la salud y también en el establecimiento de un mejor vínculo entre médico, paciente y familiares. Palabras clave: Comunicación en salud; Relaciones médico-paciente;; Terapia centrada en la persona.

\section{Introdução}

A existência da humanidade somente foi possível por meio da comunicação. À medida que crescemos, a nossa comunicação adquire maior complexidade de domínio de linguagem, leitura, análise do mundo e de si próprio (Silva, 2002). Neste processo, percebe-se que quando se estabelece uma comunicação com o outro, atribuímos significados e interpretações próprias sobre o que nos foi dito ou expressado. É por isto que não existe comunicação totalmente objetiva, pois cada sujeito é dotado de singularidade, interpretando-a da sua maneira. Assim, podemos di- zer que a comunicação tem como funções entender o mundo e o que nos cerca, relacionar-se com os demais e transformar a si mesmo. Outra forma de comunicação que é de extrema importância é a não verbal, aquela que capta informações por meio de gestos, expressões faciais e performances, na qual é possível expressar sentimentos e emoções (Ramos \& Bortagarai, 2012). Para reconhecer a comunicação não verbal é preciso sensibilidade e atenção, isto é, atentar-se mais ao outro, seus gestos e expressões (Silva, Brasil, Guimarães, Savonitti, \& Silva, 2000; Silva, 2002).

Para que a comunicação ocorra é essencial estabelecer uma interação entre os indivíduos na qual a 
informação transmitida seja compreendida (Ramos \& Bortagarai, 2012). A forma como comunicamos é um processo dinâmico e interativo, havendo a possibilidade de socializar, transmitir sentimentos, emoções e ideias (Stefanelli \& Carvalho, 2012). O estabelecimento de uma comunicação eficaz, em que emissor e receptor da mensagem possam compreender e ser compreendidos atravessa o fazer profissional em diferentes áreas. Na saúde, o estabelecimento de uma comunicação adequada faz-se fundante no sentido de promover uma compreensão apurada acerca do que se pretende informar, como diagnósticos, formas de tratamento e cuidado, além de acolher demandas, expressar sentimentos e promover práticas de saúde que respeitem o universo de cada cliente e que também se alinhem aos pressupostos de um sistema formal de cuidado (Pinheiro, 2012).

Segundo Carneiro (2017), o processo de comunicação é fundamental no contexto da saúde, pois o paciente/cliente/usuário e a família têm o direito à informação e é dever dos profissionais informá-los acerca da situação clínica em retrato. Estabelecer uma comunicação eficaz em saúde consiste em proporcionar um diálogo com qualidade, isto é, a compreensão do que queremos expressar, e se aquela informação comunicada é compreensível pelo outro indivíduo (Silva, 2002), sendo que o manejo dessa comunicação é um desafio constante para as equipes de saúde (Rodriguez, 2014). Essa comunicação também não se restringe a ações de emissão e recebimento de informações, mas justamente de estabelecimento de um relacionamento interpessoal instaurado a partir do conteúdo que se narra e do modo como isso repercute naquele que recebe a notícia. É o caso da comunicação de más notícias, como salientado no presente estudo. Como será discutido, nesse contexto de atenção não basta emitir uma informação, sendo mister cercar-se de elementos e cuidados necessários para que se possa lidar com um conteúdo frequentemente disparador de ansiedade, dor e reflexões acerca de diversos aspectos da vida, dos processos de saúde e doença, da morte e do morrer (Cavalcante, Vasconcelos, \& Grosseman, 2017; Leal-Seabra \& Costa, 2015).

No contexto hospitalar sabemos que é dever do médico comunicar os prognósticos e procedimentos aos quais o paciente irá se submeter, bem como informar os familiares. Dentre essas informações, as comunicações de más notícias também fazem parte dos procedimentos, sendo diante desses prognósticos não favoráveis que frequentemente emergem as dificuldades dos profissionais da saúde (Carneiro, 2017). Segundo Buckman (1992), um dos pioneiros em comunicação de más notícias, uma notícia difícil se caracteriza como qualquer informação que gera sensações desagradáveis associadas aos diagnósticos e prognósticos de enfermidades. A comunicação deste tipo de informação pode alterar negativamente a expectativa do paciente/cliente/ usuário em relação ao seu futuro, o que deve ser avaliado em função de diversos aspectos existentes, como as condições clínicas do caso, a existência de recursos de enfrentamento, o contexto de cuidado, a rede de apoio, entre outros elementos de ordem subjetiva e quem nem sempre podem ser mensurados, mas sim compreendidos de modo dinâmico e em sua complexidade (Mochel, Perdigão, Cavalcanti, \& Gurgel, 2010).

Certamente a comunicação de prognósticos não favoráveis é uma das tarefas mais difíceis para a equipe de saúde, podendo levar a repercussões emocionais importantes no paciente/cliente/usuário e em sua rede de apoio. Uma preocupação comum dos médicos e de sua equipe é de como a má notícia irá afetar o indivíduo e sua reação (Borges, Freitas, \& Gurgel, 2012; Pereira, 2005). Além disto, essas repercussões também afetam os próprios profissionais, pois muitos têm receios de enfrentar as reações emocionais e físicas tanto da pessoa adoecida quanto dos familiares, sobretudo a dificuldade de administrar a situação (Pereira, 2005). É importante salientar que essas dificuldades de comunicação de más notícias apresentadas pelos médicos podem ter origem na formação acadêmica, isto porque poucas universidades valorizam o ensino da comunicação em seus currículos, ou seja, é de suma importância o investimento em métodos que capacitem tais estudantes a desenvolver e aprimorar essa habilidade (Andreoli \& Erlichman, 2008; Lech, Destefani, \& Bonamigo, 2013; Lino, Augusto, Oliveira, Feitosa, \& Caprara, 2010; Sombra Neto et al., 2017; Souza \& Santos, 2013; Souza \& Scorsolini-Comin, 2011).

Para auxiliar os profissionais de saúde a transmitirem uma notícia considerada desfavorável, foram elaborados protocolos técnicos que oferecem algumas orientações para sistematizar a transmissão de uma má notícia, tornando-a menos traumática tanto para o médico e, sobretudo, para o paciente. Esses protocolos constituem guias metodológicos para os profissionais de saúde, favorecendo a relação médico-paciente (Pereira, Fortes, \& Mendes, 2013), como detalhado a seguir.

\section{Protocolos para a comunicação de más notícias}

Em 1992, Bachman publicou o protocolo SPIKES com a intenção de tornar mais didática a comunicação de más notícias (Buckman, 1992; Sombra Neto et al., 2017). O protocolo SPIKES (Setting; Perception; Invitation; Knowledge; Explore emotions; Strategy and sumary) surgiu com o objetivo de habilitar o médico e a equipe multiprofissional a comunicarem más notícias para pacientes com câncer (Baile et al., 2000). Tal protocolo é composto por seis passos: (1) cuidado com o local onde será feita a revelação da notícia; (2) percepção do paciente em relação a própria condição e gravidade; (3) apresentação de possibilidades de tratamento; (4) abordar e reconhecer as emoções e sentimentos, agindo de maneira empática; (5) Abordar as emoções dos pacientes após a comunicação da má noticia; (6) finalização, que consiste em realizar uma síntese de tudo o que foi dito (Baile et al., 2000; Buckman, 1992). A literatura científica tem reconhecido o SPIKES como um protocolo útil justamente por ser didático e explorar os principais pontos referentes à comunicação.

No protocolo CLASS, a metodologia para comunicar uma notícia difícil é constituída por cin- 
co passos. A primeira etapa é considerar o contexto físico, ou seja, priorizar um ambiente adequado para a conversa; o segundo passo visa à habilidade de escuta, isto é, o profissional deve estar disponível para escutar o paciente; posteriormente é preciso reconhecer as emoções e explorá-las de maneira empática; a quarta etapa é traçar um planejamento de estratégias, descrevendo com clareza a proposta terapêutica e seus procedimentos; por fim, realiza-se uma síntese dos principais tópicos discutidos durante a conversa, verificando se há dúvidas (Albert Einstein Instituto Israelita de Ensino e Pesquisa, s/d).

Outro protocolo frequentemente referido na literatura é nomeado como P-A-C-I-E-N-T-E e foi elaborado por Pereira (2010), baseado no protocolo de comunicação SPIKES e adaptado para a realidade brasileira. Este protocolo é constituído por sete etapas: $\mathbf{P}$ - Prepare-se, consiste em checar as informações a serem comunicadas aos pacientes, além de estar em um ambiente físico com privacidade e conforto; $\mathbf{A}$ Avalie o quanto o paciente sabe e o quanto quer saber; C - Convite à verdade; I - Informe, isto é, compartilhe a informação em quantidade, velocidade e qualidade suficiente para que o paciente faça sua decisão; E Emoções, consiste em dar espaço ao paciente em expressar suas emoções; $\mathbf{N}$ - Não abandone o paciente, ou seja, assegurar-se que o paciente receberá acompanhamento médico; T - Trace uma Estratégia, em suma, significa planejar os próximos cuidados a serem oferecidos e opções de tratamento (Pereira, 2010). Segundo o estudo realizado por Pereira, o protocolo foi considerado prático e útil, sendo que os participantes consideraram a etapa de não abandonar o paciente e a etapa de lidar com as emoções do paciente as mais difíceis contidas no instrumento.

Como podemos observar, esses protocolos possuem muitos pontos de semelhanças, sendo que todos se baseiam no pressuposto básico da necessidade de sistematizar a comunicação quando se trata de uma má notícia. Entre os pontos priorizados nesses instrumentos destacamos a necessidade de assertividade por parte do profissional que comunica a má notícia, notadamente o médico, e também o estabelecimento de um relacionamento interpessoal de confiança e segurança. A fim de explorar alguns desses aspectos suscitados por essas metodologias, neste estudo aproximaremos esses principais protocolos de comunicação em saúde presentes na literatura (SPIKES, CLASS e P-A-C-I-E-N-T-E) com as atitudes/ condições facilitadoras contidas na abordagem centrada na pessoa (ACP), elaborada por Carl Rogers. As principais condições abordadas neste artigo serão as de autenticidade/congruência, consideração positiva incondicional e compreensão empática (Rogers, 1973), conforme relatado a seguir.

\section{As atitudes/condições facilitadoras no estabelecimento da comunicação de más notícias: uma aproximação com a Abordagem Centrada na Pessoa (ACP)}

A ACP foi desenvolvida por Carl Rogers, nos Estados Unidos, e se insere na chamada terceira força da Psicologia, ou Psicologia Humanista. Ro- gers traz para a psicoterapia uma visão positiva do homem, na qual o ser humano possui liberdade e poder de escolha, mostrando-se um agente criativo na realidade que o rodeia. Sua teoria passou por diversas fases e suas formulações começaram a partir de modelos tradicionais de clínica, estendendo-se para as ideias sobre relacionamentos interpessoais, visando ao crescimento psicológico independentemente do contexto em que as pessoas estivessem inseridas (Bezerra \& Bezerra, 2012). Até constituir a ACP, em um momento de consolidação de suas ideias, Rogers empregou diferentes nomenclaturas para o conjunto de suas propostas, como Psicoterapia Não-Diretiva, Aconselhamento Não-Diretivo, Terapia Centrada no Cliente, Ensino Centrado no Aluno e Liderança Centrada no Grupo. A denominação ACP foi oficializada em 1977 (Moreira, 2010), constituindo um referencial não mais aplicável exclusivamente ao contexto psicoterápico e à relação terapeuta-cliente, mas a uma gama de possibilidades no estabelecimento de relações interpessoais, a exemplo daquelas vivenciadas no campo do cuidado em saúde e também nos diferentes cenários educacionais.

Por meio da noção de tendência atualizante, ou seja, de uma tendência inerente de desenvolver-se em uma direção positiva, a ideia central da ACP é de que o indivíduo possui "dentro de si, vastos recursos para a auto compreensão, para alterar seu autoconceito, sua atitude e seu comportamento, e que tais recursos podem ser liberados quando se conta com determinado clima psicológico" (Almeida, 2009, p. 180), ou seja, quando existem atitudes psicológicas facilitadoras. A partir disso, Rogers trouxe para o campo psicoterápico a ideia das condições facilitadoras e das atitudes que um terapeuta deveria ter para possibilitar uma mudança no processo terapêutico. Entre as seis condições encontramos, por exemplo, a necessidade de que haja contato psicológico entre cliente (que deve estar em estado de incongruência) e terapeuta (que deve estar congruente ou integrada na relação), devendo o terapeuta experienciar e comunicar ao cliente a sua consideração positiva incondicional e compreensão empática. Entre essas condições encontram-se as três atitudes facilitadoras propostas por Rogers: autenticidade/congruência, consideração positiva incondicional e postura empática (Fontgalland \& Moreira, 2012).

Em termos da caracterização dessas atitudes, pode-se compreender que a autenticidade, também conhecida como congruência, envolve a necessidade de estar congruente consigo mesmo, integrado e harmônico, aberto aos próprios sentimentos, podendo escutá-los, elaborá-los e expressá-los. Ser congruente/autêntico envolve estabelecer uma correspondência entre o que o terapeuta experiencia e o que ele comunica ao cliente, sendo ele mesmo nessa relação (Moreira, 2010). Neste sentido, quanto mais o terapeuta se conhece e se ouve, mais apto ele está para ouvir o "ser" do outro. A atitude facilitadora de congruência quer dizer que o profissional deve ser o mais próximo possível do que é em suas relações, inclusive no encontro com o cliente, onde 
seja possível estar integralmente a serviço de ajudar e, consequentemente, coerente com o seu modo de ser, pensar, agir e de se relacionar (Rogers, 1977; Scorsolini-Comin, 2015).

A consideração positiva incondicional pode ser compreendida como o respeito incondicional, por parte do terapeuta, à individualidade do cliente (Moreira, 2010). Em outras palavras, refere-se ao "fato de o profissional acreditar profundamente que aquela pessoa em busca de ajuda tem condições de amadurecer e resolver seus conflitos a partir desse crescimento e da potencialização de suas capacidades. É a aceitação do cliente sem reservas, julgamentos ou questionamentos sobre o seu modo de ser e as escolhas que tem tomado em sua vida" (Scorsolini-Comin, 2015, p. 57).

A compreensão empática é caracterizada pela atitude de experimentar o que o outro está sentindo por meio de uma condição de "como se" estivesse no lugar dele, enxergando através da perspectiva do mesmo (Rogers, 1973). Por meio da empatia, o terapeuta busca perceber e compreender o mundo na perspectiva do cliente (Moreira, 2010), o que promoveria não apenas uma maior proximidade, como contribuiria na aceitação do cliente sem julgamentos, compreendendo sua forma de ser e experienciar o mundo.

Essas três atitudes, priorizadas no presente estudo, seriam consideradas necessárias e suficientes para que o processo terapêutico ocorresse e obtivesse êxito (Scorsolini-Comin, 2015), o que posteriormente foi expandido para pensarmos as relações interpessoais de ajuda que podem ocorrer em settings não terapêuticos, como em escolas e espaços de cuidado em saúde, como hospitais, por exemplo (Trzan-Ávila \& Vilela, 2012). É nesse cenário de expansão da ACP e do seu emprego para além da psicoterapia que o contexto de atenção à saúde passou a se apropriar desses conhecimentos, sobretudo em relação à oferta de uma escuta sensível e que ajude o paciente/cliente/usuário/família a entender e enfrentar o momento de enfermidade, tradicionalmente associado ao sistema de cuidado formal representado em equipamentos como os hospitais (Espinha, 2007). A partir desses pressupostos, considera-se que a comunicação de más notícias, uma competência de difícil manejo no cuidado em saúde (Andreoli \& Erlichman, 2008; Lech et al., 2013; Lino et al., 2010; Sombra Neto et al., 2017), pode ser compreendida como uma prática que pode se beneficiar de uma leitura em termos das atitudes facilitadoras propostas por Rogers.

A partir desse panorama, este estudo teve por objetivo realizar uma aproximação teórica das atitudes facilitadoras descritas por Rogers (autenticidade/congruência, consideração positiva incondicional e empatia) com os três protocolos de comunicações de más notícias mais encontrados na literatura médica (SPIKES, CLASS e P-A-C-I-E-N-T-E). Este estudo mostra-se relevante do ponto de vista científico por levantar questões a respeito de uma maior informação e humanização dos profissionais da saúde em relação ao comunicar uma má notícia, haja vista que a maneira como isso se processa pode afetar a compreensão dos pacientes/clientes/ usuários a respeito da notícia e, consequentemente, a forma de aderir ao tratamento (Monteiro \& Quintana, 2016) e prosseguir com os cuidados em saúde.

\section{Método}

\section{Tipo de estudo}

Trata-se de um estudo teórico e documental delineado a partir de uma revisão integrativa da literatura científica, método este que pressupõe a busca, seleção e sistematização de informações acerca de determinado tópico com vistas à sumarização do conhecimento produzido (Scorsolini-Comin, 2014), sendo uma estratégia para a prática baseada em evidências (Mendes, Silveira, \& Galvão, 2008). Classicamente, as revisões integrativas são desenvolvidas em diferentes etapas, pressupondo a inclusão de materiais heterogêneos em termos de evidências, ou seja, utilizando estudos com maior ou menor evidência decorrentes de investigações realizadas sob diversas metodologias. As etapas para a confecção da revisão integrativa sumarizadas pela literatura (identificação da questão de pesquisa, estabelecimento de critérios de busca, seleção e incorporação de estudos, avaliação e interpretação dos resultados) foram customizadas na presente investigação, a fim de cotejar os objetivos apregoados. A fim de empregar estudos disponíveis em meios eletrônicos e com fácil e rápida atualização, foram selecionados apenas artigos científicos, como também sugerido nas revisões integrativas do campo da saúde (Mendes et al., 2008).

A busca dos artigos nas bases de dados/bibliotecas ocorreu em três momentos. Na primeira etapa houve a intenção de procurar artigos que abordassem a temática da comunicação de más notícias. Na segunda etapa da pesquisa, o foco estava nos protocolos utilizados para comunicar más notícias. No terceiro momento, pesquisou-se artigos referentes às condições facilitadoras proposta por Carl Rogers na abordagem centrada na pessoa.

\section{Procedimentos de busca e análise de dados}

Primeira etapa de busca e seleção. As bases de dados/bibliotecas utilizadas para a pesquisa foram: LILACS (Literatura Latino Americana e do Caribe em Ciências de Saúde), SciELO (Scientific Electronic Library Online), PePSIC (Periódicos Eletrônicos em Psicologia) e MEDLINE (Medical Literature Analysis and Retrieval System Online). Os seguintes descritores foram empregados: "Comunicação em Saúde" and "Más notícias" nas bases de dados LILACS, SciELO e PePSIC, enquanto na base MEDLINE utilizou-se palavra-chave em inglês "Communicating bad news". A busca foi realizada no segundo semestre de 2017, sem delimitação temporal, pois a intenção foi analisar a produção científica acerca do tema ao longo dos anos. Como critérios de inclusão considerou-se artigos na íntegra e que tivessem relação com a comunicação de más notícias no contexto da saúde. No que diz res- 
peito às bases de dados LILACS, SciELO e PePSIC, encontrou-se um total de 228 artigos. Após a aplicação dos critérios de inclusão restaram 16 artigos. Já na base de dados MEDLINE, a busca resultou em um total de 43 artigos e, aplicando os critérios de inclusão, foram selecionados seis artigos. Portanto, no que diz respeito à pesquisa ao tema de comunicação em saúde, foram recuperados um total de 22 artigos.

Segunda etapa de busca e seleção. Para as pesquisas dos protocolos SPIKES, CLASS e P-A-C-I-E-N-T-E, as buscas ocorreram no Google Acadêmico, em livros, e em materiais concedidos através de cursos sobre como comunicar más notícias. Ressalta-se que o protocolo SPIKES é o mais difundido entre os três, sendo que cinco estudos sobre esse protocolo comporão o presente corpus. No que diz respeito ao protocolo CLASS, foram incorporados dois estudos e outros três que abordaram o P-A-C-I-E-N-T-E. Assim como na primeira etapa não houve delimitação temporal, haja vista a intenção de analisar a produção científica acerca do tema ao longo dos anos.

Terceira etapa de busca e seleção. As três condições/atitudes facilitadoras de Carl Rogers foram pesquisadas separadamente, mas todas elas vinculadas à palavra-chave "Abordagem Centrada na Pessoa". A pesquisa foi realizada nas bases/bibliotecas LILACS, SciELO, Google Acadêmico e em livros. Foram incorporados ao estudo um total de 10 referências sobre o tema.

Análise do corpus. O corpus analítico delimitado após essas três etapas foi lido, categorizado e dividido para apresentação a partir das três atitudes facilitadoras elencadas a priori: (a) $1^{\mathrm{a}}$ atitude: autenticidade/congruência; (b) $2^{\text {a }}$ atitude: consideração positiva incondicional; (c) $3^{\mathrm{a}}$ atitude: compreensão empática. Essa divisão em categorias cumpre uma função didática para a melhor visualização dos aspectos retratados nesse estudo, mas deve-se explicitar que a análise integrada dessas três atitudes deve ser buscada a todo o momento, haja vista que as mesmas ocorrem de modo fluido e contínuo em todo processo terapêutico e, consequentemente, no estabelecimento de um relacionamento interpessoal com foco no cuidado, como retratado no presente estudo. Essas categorias foram interpretadas a partir da Abordagem Centrada na Pessoa, empregando tanto estudos de Rogers $(1973,1977,2012)$ como de alguns de seus comentadores (e.g. Fontgalland \& Moreira, 2012; Moreira, 2010). A interpretação também ocorreu a partir da literatura científica sobre os protocolos de comunicação de más notícias em contextos de atenção à saúde (e.g. Burlá, 2006; Cruz \& Riera, 2016; Petrilli, Pascalicchio, Dias, \& Petrilli, 2000; Pereira, 2010). A discussão dos principais achados será apresentada a seguir.

\section{Resultados e Discussão}

Primeira atitude facilitadora no processo de comunicação de más notícias: autenticidade/congruência

Como destacado na introdução deste estudo, as proposições de Rogers quanto às atitudes facilitadoras tinham como cenário a relação terapêutica ou a situação de contato psicológico estabelecida entre terapeuta (que deve estar congruente e integrado) e cliente (que deve se encontrar em um estado de incongruência). Como a ACP foi se expandindo e compondo um repertório de conhecimentos desenvolvidos em cenários educacionais e de saúde para além da clínica tradicional, a nomenclatura proposta nessa análise tomará por base a figura do profissional de saúde que comunica a má notícia (e.g. médico) como sendo equivalente a do terapeuta e a do paciente/cliente/usuário de um equipamento de saúde como próxima da descrição de cliente feita por Rogers $(1973,1977)$.

Em um contexto hospitalar, por exemplo, se substituirmos a figura do terapeuta pela do médico, este deverá estabelecer um encontro de ajuda para com o outro, no caso, o paciente/cliente/usuário. Nesse sentido, quando o médico tem a função de comunicar uma má notícia ao paciente e seus familiares, a maioria desses profissionais sente dificuldade em estabelecer o diálogo. A dificuldade em comunicar más notícias, relatada por Victorino et al. (2007), vem à tona pelos próprios medos médicos, isto é, o receio de causar dor ao paciente, de não se sentir seguro em "passar" a notícia, de ser culpado pelo paciente e familiares, de ter algum problema judicial, do desconhecimento, de expressar as emoções, e também o óbito do paciente.

Segundo Lino et al. (2010), assim como é difícil para o paciente receber a notícia, o ato de transmitir uma notícia desagradável é desconfortável também para o médico por vários motivos. Primeiramente, o médico/terapeuta se vê na situação difícil de lidar com emoções experimentadas pelo paciente/cliente e suas reações. Frente a esta situação, o médico/terapeuta tem a possibilidade de proporcionar ajuda por meio da sua congruência. Ou seja, ao vivenciar sentimentos desfavoráveis diante do paciente/cliente, o médico/terapeuta tem acesso a seus sentimentos de uma forma consciente, estabelecendo um encontro direto com o paciente/cliente, de pessoa para pessoa. Consequentemente, o advento de tal contato significa que o médico/terapeuta está permitindo-se "ser o que se é", portanto, sendo congruente (Rogers \& Stevens, 1978).

Obviamente que vivenciar essa atitude não parte apenas de um desejo do médico ou de outros profissionais de saúde, de modo automático e perene. Ser/estar congruente/integrado parte de um processo psicológico desenvolvido/vivenciado pelo profissional de saúde, um trabalho que requer dedicação, tempo, disponibilidade interna e busca pelo autoconhecimento, o que muitas vezes passa pela existência de processos de cuidado que extrapolam o contexto laboral, como a realização de psicoterapia pessoal. Também as experiências prévias com esse tipo de comunicação e desfechos positivos em termos da efetividade desses diálogos podem ser importantes para dotar o profissional de maior segurança e, consequentemente, ajudá-lo em seu processo de tornar-se congruente. Para experienciar essa congruência/autenticidade, é necessário que 
o profissional de saúde reflita sobre a sua atuação, rompendo com os paradigmas biomédicos que pressupõem a necessidade constante de controle sobre os processos, relativizando o alcance do seu saber técnico e buscando uma postura mais humanizada no contato com o outro. E importante destacar que ninguém atinge totalmente esta condição. Contudo, quanto mais o terapeuta, no caso o profissional de saúde, é capaz de ouvir e aceitar o que ocorre em seu íntimo, e quanto mais é capaz de, sem medo, ser a complexidade de seus sentimentos, maior é o grau de sua congruência.

É possível notar que a ideia central da teoria rogeriana de congruência/autenticidade está presente em algumas etapas dos protocolos SPIKES, P-A-C-I-E-N-T-E e CLASS. Tal ideia corresponde às etapas $\mathbf{S}$ e I do protocolo SPIKES, presentes também nas etapas $\mathbf{P}$ e A do protocolo P-A-C-I-E-N-T-E e, por fim, na fase C, do protocolo CLASS. Em geral, as etapas que fazem uma associação com a autenticidade são as primeiras sugeridas pelos protocolos. Isto vem do fato de que todas relacionam-se ao cuidado do profissional de preparar-se para o encontro, preocupando-se com o setting onde a conversa irá ocorrer, bem como estabelecer um bom vínculo com o paciente (Cruz \& Riera, 2016; Baile et al., 2000; Pereira, 2010).

Esses protocolos, no entanto, focam-se na relação interpessoal que se estabelece no presente e que se realiza no processo de comunicação de uma má notícia. Essa preparação do setting, por exemplo, representa apenas o final de um processo que envolve a busca pela maior integração do profissional. Assim, pressupõe-se que o processo de preparação para o estabelecimento de uma relação de ajuda é o que contribui para uma maior autenticidade/congruência, o que promoveria, na atuação prática, a possibilidade de estar integrado, inteiro e capaz de informar o que precisa ser comunicado. Essa disponibilidade de compartilhar com o outro o que se é, o seja, demonstrando sua integração, permitiria o estabelecimento de um vínculo que favoreceria a comunicação e, provavelmente, as repercussões após a notícia.

É importante dizer que nas fases I do protocolo SPIKES e A e $\mathbf{C}$ do protocolo P-A-C-I-E-N-T-E o profissional é orientado a fornecer informações sobre seu diagnóstico, convidando-o para a verdade, diretamente associada a uma postura de integração. A integração, nesse caso, não está relacionada a um desfecho positivo necessariamente (cura, melhora, restabelecimento da saúde, por exemplo), mas à possibilidade de poder comunicar o que é necessário, a fim de que determinadas ações possam ser colocadas em prática, como a realização de determinados tratamentos de caráter curativista ou paliativista, a depender do caso.

No contexto da comunicação em saúde, os protocolos têm destacado que é importante avaliar o quanto o paciente sabe, e o quanto quer saber sobre a notícia, sendo de total direito optar por não saber sobre a informação (Cruz \& Riera, 2016; Pereira, 2010). Aqui não se trata, portanto, de assumir um compromisso exclusivamente com a verdade (a notícia que se tem para comunicar), mas com o próprio paciente/cliente/usuário, respeitando a sua decisão de saber ou não. Esse aspecto nos conduziria, analiticamente, para elementos mais relacionados à empatia, por exemplo, como destacaremos na terceira categoria, mas é importante considerar que o profissional autêntico/congruente, justamente por estar integrado, consegue lidar com a incongruência de seu cliente, possivelmente em estado de ansiedade devido ao quadro de adoecimento. Ao reconhecer e respeitar a incongruência do outro, consegue prestar um cuidado mais autêntico.

Essas orientações vão ao encontro das ideias de Rogers de ser congruente, tanto para o médico, quanto para o paciente. $\mathrm{O}$ médico também precisa assumir ou ter consciência de seus sentimentos por vezes negativos perante o paciente e à necessidade de comunicar notícias ruins, entrar em contato com as suas emoções pessoais, suas dificuldades com determinadas abordagens e assuntos, reconhecendo limitações que podem ser trabalhadas em diferentes espaços, dentro e fora do seu setting profissional. A partir dessa leitura, não podemos compreender que, para o médico, por exemplo, o ser autêntico é estritamente fornecer a verdade para o paciente, não escondendo informações e, sobretudo, deixando transparecer o "ser o que se é". Ser autêntico pode ser estar junto do outro oferecendo sua integração, aceitando as decisões que podem ou não ser compatíveis com seu esquema de referência. Este aspecto emerge como intimamente ligado à segunda atitude descrita por Rogers e explicitada na categoria seguinte. Finalizando a primeira categoria, deve-se sublinhar que o processo de tornar-se autêntico/congruente deve ser constante, convidando os profissionais de saúde para um trabalho de desenvolvimento pessoal que tome por base esse contexto de atuação fortemente estressor e potencializador de sentimentos por vezes de difícil manejo (Andreoli \& Erlichman,2008; Lech et al., 2013; Sombra Neto et al., 2017).

Segunda atitude facilitadora no processo de comunicação de más notícias: a consideração positiva incondicional

A ideia de Rogers sobre a consideração positiva incondicional pelo outro também pode ser depreendida nos três protocolos, mais especificamente nas etapas I e $\mathbf{S}$ do SPIKES, I e E do protocolo P-A-C-I-E-N-T-E, como também nas duas últimas etapas do protocolo CLASS, $\mathbf{S}$ e $\mathbf{S}$. No que diz respeito às etapas do protocolo SPIKES, na letra I - (convidando para o diálogo), o profissional da saúde identifica se o paciente sabe o que está acontecendo, se gostaria de ser totalmente informado, ou até mesmo, se prefere que algum membro da família tome decisões por ele (Albert Einstein Instituto Israelita de Ensino e Pesquisa, s/d). Na etapa $\mathbf{S}$ (Estratégia e Síntese) ocorre o esclarecimento das estratégias terapêuticas traçadas e a realização da síntese do encontro, na qual é importante deixar claro para o paciente que ele não será abandonado, que existe um plano ou tratamento, curativo ou não (Cruz \& Riera, 2016).

Ao perguntar ao paciente/cliente/usuário se ele 
quer ser informado ou se deseja que outra pessoa receba essas informações pressupõe-se a aceitação de que a pessoa pode fazer escolhas relacionadas à sua própria vida e aos seus processos de saúde e doença. Permitir que a mesma escolha ser comunicada ou não é respeitar a sua capacidade de decidir, de se posicionar, de ser ativa mesmo em um momento no qual possa estar mais fragilizada ou demandando cuidados especiais. A situação de adoecimento potencializa a desintegração, de modo que acreditar que a pessoa pode decidir (o que novamente não se relaciona à necessidade de um desfecho positivo de cura, por exemplo) é considerá-la de modo positivo, reforçando sua tendência atualizante. O quadro de adoecimento deve ser compreendido como um contexto desenvolvimento que pode ser situacional ou permanente, mas que não pode se sobrepor à dimensão da pessoa.

Nas duas etapas finais do protocolo P-A-C-I-E-N-T-E também é possível notar que há uma preocupação em discutir os próximos passos do paciente. Neste protocolo (especificamente nas etapas $\mathbf{T}$ e E) é sugerido estabelecer um contato mais permanente com o paciente, isto porque a estratégia de comunicação entre médicos e pacientes facilita a informação de diagnóstico e prognóstico de uma forma sistemática e verdadeira, respeitando a autonomia e individualidade. Tanto os profissionais da saúde quanto os pacientes beneficiam-se de uma relação transparente em que a vontade do paciente é priorizada (Pereira, 2010), o que reforça os pressupostos da condição de consideração positiva incondicional. Ao empregarmos o adjetivo incondicional, a exemplo do que sugere Rogers, não estamos nos comprometendo a uma aceitação fantasiosa, acrítica ou que não corresponda à verdade, mas a uma crença no potencial de crescimento, amadurecimento e integração da pessoa, o que pode ser favorecido e potencializado pelas ações dos profissionais de saúde nesse contexto de atenção. Essa aceitação não pode estar condicionada aos possíveis desfechos do adoecimento, mas a uma atitude que deve ser desenvolvida tendo como referência a pessoa em busca de sua integração e congruência, ou seja, que desvela a sua tendência atualizante.

Cada indivíduo carrega consigo marcas pessoais que fundamentam suas emoções, motivações e atitudes. Assim sendo, dar uma má notícia é o mesmo que abranger os diferentes estágios de suas emoções e sentimentos (Burlá, 2006). Kübler-Ross (2008) afirma que toda perda, caracterizada pelo luto, passará por pelo menos dois estágios das cinco fases do luto (Negação, Raiva, Barganha, Depressão e Aceitação). Portanto, entender e identificar esses momentos que o indivíduo está enfrentando é essencial ao comunicar uma má notícia.

Como explicitado, essas etapas apresentam muito das características de consideração positiva incondicional que, segundo Rogers (1973), é sentir uma calorosa preocupação pelo seu cliente/paciente, demonstrando uma atmosfera de "eu me preocupo". Ou seja, é importante que o médico tenha o cuidado de pensar em como comunicar a notícia e os impactos que ela pode gerar na vida desta pessoa
(Pinheiro, 2012). No mais, quando o profissional traça uma estratégia de tratamento, alcançando as necessidades do paciente e, sobretudo, respeitando suas escolhas, é uma forma de preocupação com a vida do paciente. O delineamento dessa estratégia no cuidado em saúde ainda é prioritariamente construído pelo profissional, em detrimento à elaboração, por parte da dupla profissional-cliente, de ações e metas para o cuidado. Em uma leitura rogeriana, haveria a necessidade de que tais decisões partissem de ambos os lados, ampliando as possibilidades de escuta sobre as necessidades do cliente nesse cenário.

Podemos dizer que para a consideração positiva incondicional ocorrer nestas etapas, o profissional de saúde deve tanto acolher as decisões e dúvidas do paciente/cliente/usuário/família, como também suas respostas emocionais (raiva, negação, etc.), estando sempre disponível para o diálogo. Independentemente de qual seja a decisão do paciente, acreditar neste sujeito e em sua capacidade de mudança deve ser uma crença capaz de atuar no estabelecimento da confiança apesar dos rumos do tratamento. Este modo de aceitação equivale a acolhê-lo como pessoa (Scorsolini-Comin, 2015), corporificando os pressupostos da ACP.

Terceira atitude facilitadora no processo de comunicação de más notícias: a compreensão empática

Realizando uma aproximação desta atitude empática com os protocolos, percebe-se que nas etapas K (Dando Conhecimento e Informação ao Paciente) e $\mathbf{E}$ (Abordar as emoções) do protocolo SPIKES, como também as etapas $\mathbf{E}$ (emoções) e $\mathbf{N}$ (não abandonando o paciente) do protocolo P-A-C-I-E-N-T-E e L (Habilidade de escutar) e A (Reconhecer emoções e explorá-las) do protocolo CLASS abordam e sugerem posturas mais empáticas do profissional perante a comunicação de más notícias.

Nas etapas referentes ao protocolo SPIKES o profissional da saúde irá contextualizar e avisar o paciente que más notícias estão por vir. Avisá-lo dessas notícias pode diminuir o impacto que elas podem causar (Baile et al., 2000). Recomenda que a linguagem utilizada pelo médico seja compreensível pelo paciente, bem como o cuidado com sua fala, evitando ser "duro" (exemplo: "Você tem um câncer muito agressivo e a menos que inicie o tratamento imediatamente você vai morrer") (Baile et al. 2000). O lidar com as emoções do paciente é considerada a tarefa mais difícil da transmissão de más notícias, o profissional pode oferecer apoio e solidariedade ao paciente com uma resposta afetiva (Baile et al. 2000). As fases $\mathbf{E}$ e $\mathbf{N}$ do protocolo P-A-C-I-E-N-T-E, como também as etapas L e A do protocolo CLASS, vão ao encontro das duas etapas do protocolo SPIKES mencionada anteriormente. Ambas as etapas, A do CLASS e $\mathbf{E}$ do protocolo P-A-C-I-E-N-T-E têm como objetivo explorar as emoções, reconhecer e amparar as emoções dos pacientes. $\mathrm{O}$ diferencial é que na etapa $\mathbf{N}$ do protocolo P-A-C-I-E-N-T-E sugere que o profissional se comprometa com o paciente e não o abandone durante o tratamento (Pereira, 2010). 
Todas estas etapas aproximam-se da atitude empática proposta por Rogers, pois cabe ao profissional ser sensível aos sentimentos e às reações pessoais que o cliente experiencia em cada momento (Fontgalland \& Moreira, 2012). Para estarmos com o outro empaticamente é necessário "penetrar no mundo perceptual do outro e sentir-se totalmente à vontade dentro dele" (Rogers, 1977, p. 73). Obviamente que o acesso ao mundo do outro não ocorre de modo automático nem utilitário, mas a partir da construção de um sólido repertório de atitudes para o estabelecimento de relacionamentos interpessoais pautados na necessidade de reconhecer o outro como um sujeito que pensa, sente, age e reage de modos distintos. A capacidade de reconhecer no outro tanto proximidades quanto distanciamentos pode ser um convite para o desenvolvimento da empatia, recuperando a noção de que, para compreender o outro, é importante conhecê-lo e situá-lo diante de um contexto de referência próprio. Ao lançar esse olhar para a alteridade, inicia-se o processo de compreensão de que aquela pessoa se estrutura de um modo que deve ser reconhecido para ser aceito e disparar a adoção de estratégias para melhor manejo de tais modos de ser e de viver.

Nas duas últimas etapas existentes no protocolo CLASS há também uma proposta de estratégia (S) e uma síntese (S), como apresentados nos demais protocolos. No item estratégia $\mathbf{S}$ é sugerido que o profissional pense o que é melhor em termos médicos, considere as expectativas do paciente quanto às condições emocionais, sociais e econômicas, propondo uma estratégia e dando ênfase à qualidade de vida. Ainda na etapa $\mathbf{S}$, é necessário levar em consideração a resposta do paciente (prestando atenção ao estágio que ele está: raiva, negação, barganha, por exemplo), na qual é importante esboçar um plano, descrevendo com clareza a proposta terapêutica, a sequência dos exames, retornos, etc. Na etapa de síntese - $\mathbf{S}$ é recomendado envolver o término da entrevista com o paciente com três componentes principais: um resumo dos principais tópicos discutidos, o questionamento ao paciente se existe algum tópico que gostaria de discutir e, por fim, realizar um roteiro claro para o próximo encontro (Albert Einstein Instituto Israelita de Ensino e Pesquisa, s/d; Petrilli et al., 2000).

Esses aspectos retomam o estabelecimento da compreensão empática na comunicação e na adoção de tratamentos, por exemplo. Ao buscar compreender a fase na qual o paciente/usuário/cliente se encontra, por exemplo, apresenta-se a possibilidade de tentar entender o modo como essa pessoa tem agido, reagido e se colocado diante do que precisa ser comunicado. Esse exercício é potente no sentido de sugerir tratamentos, formas de cuidado ou mesmo prescrições médicas que sejam condizentes com o que o cliente está preparado para suportar e, mais do que isso, com o seu grau de integração diante do cenário. A empatia permite que o profissional de saúde justamente se coloque no lugar desse paciente/cliente/usuário e reconheça as necessidades e potencialidades do mesmo para o cuidado.

\section{Síntese analítica}

É possível observar nesses protocolos uma preocupação em diminuir os impactos negativos gerados no paciente diante a revelação de uma notícia ruim. As evidências mostram que a atitude do profissional e a capacidade de comunicação desempenham um papel fundamental e decisivo no modo que o paciente enfrentará seu problema (Victorino et al., 2007). O que está ao alcance do profissional, diante disso, é dar subsídios humanos por meio do acolhimento, para que a família se sinta principalmente acolhida diante a má notícia. Sendo assim, é necessário exercer uma postura pautada nos princípios éticos como o do sigilo e do respeito para com a pessoa em sofrimento. Embora o indivíduo possa se lembrar com pesar do quão difícil foi passar por este momento de luto, pode também rememorar a confiança e o acolhimento recebidos na ocasião (Azeredo, Rocha, \& Carvalho, 2011).

As formas como as etapas dos protocolos foram desenvolvidas contribuem para a uma atitude mais empática do profissional. Isto é, quando é sugerido por parte desses protocolos checar se o paciente está compreendendo o que está sendo dito, como também de não o abandonar no percurso do seu tratamento, o profissional é convidado a estar junto com o outro, de mergulhar no universo do paciente, compreendendo de forma vivencial as dores e dificuldades de se receber uma má notícia. Ao colocar em prática essa atitude e as demais elencadas nessa análise, consegue-se possibilitar um cuidado mais humanizado e integrado, respondendo de modo adequado às orientações para o cuidado em saúde veiculadas por órgãos profissionais e instituições de formação na contemporaneidade.

Percebe-se que comunicar más notícias é uma das tarefas mais difíceis e complexas que os profissionais de saúde têm que enfrentar, gerando forte impacto psicológico no paciente e sua rede de apoio. Por isso, quem recebe uma má notícia dificilmente esquece onde, como e quando ela foi comunicada (Victorino et al., 2007). A comunicação com os pacientes e/ou familiares deve ser vista como uma oportunidade única de ajuda perante aos momentos críticos, considerando como parte do tratamento.

Os protocolos foram criados justamente para estabelecer um melhor vínculo e, sobretudo, uma comunicação eficiente com os pacientes e familiares. Diante da análise feita entre a aproximação dos conteúdos e fases destes protocolos com as três atitudes facilitadoras propostas por Rogers, percebe-se que estas condições dão a oportunidade de os profissionais refletirem o modo e como estão estabelecendo/conectando-se com o paciente. Provavelmente dispor de um protocolo de atenção ao usuário no hospital facilitará a comunicação. No entanto, é importante ressaltar que cada paciente e família fazem parte de um mundo diferente (o mundo de seus sentimentos) e sua história tem que ser considerada.

É pela linguagem que fazemos as descobertas e conhecemos o mundo do outro e com o outro. Assim, o profissional que consegue ser compreendido 
pelo paciente estabelece com ele e seus familiares uma relação empática, criando um relacionamento permissivo, envolto de um clima afetivo, de respeito e responsabilidade. Quanto mais o profissional conseguir ser genuíno, real, apresentando-se como tal, mais útil e acolhedora será a relação. Mais do que localizar tais atitudes nos protocolos de comunicação de más notícias é mister integrar esses aspectos à formação de profissionais em saúde, haja vista que compõem um repertório que pode ser empregado em diversas situações da assistência em saúde, melhorando a qualidade do atendimento prestado e promovendo uma escuta e um acolhimento humanizado, assim como preconizado na contemporaneidade. Tal integração, embora seja fomentada, ainda constitui um desafio a ser considerado na atenção em saúde.

Contudo, é necessário resgatar a importância da relação equipe-família-paciente como prática diária, haja vista que quando há uma capacidade por parte do profissional em escutar, compreender e de exercer a empatia, gera o resgate de uma relação mais humana que ficou perdida na ciência. Assim, através da humanização o paciente é visto e tratado como pessoa, deixando de ser caracterizado apenas por números e patologias. Então, para que tal relação seja mais humana, o diálogo entre profissionais e pacientes é de extrema importância, pois quando o paciente é colocado em uma posição ativa na construção do próprio tratamento, bem como procura saber o que ocorre com ele, estabelece uma parceria entre paciente e profissional. Isto é possível quando ambos se tornam parceiros e juntos traçam os objetivos do tratamento (Souza \& Santos, 2013).

\section{Considerações Finais}

Podemos afirmar que a boa comunicação é aquela que proporciona a diminuição de conflitos e mal-entendidos entre equipes de saúde, paciente e familiares. Portanto, é sobre o paciente e a família que incidem as consequências de um ambiente relacional e de uma comunicação ruim, na medida em que são mal atendidos. A qualidade da comunicação é construída. Quando o médico tem a tarefa de comunicar uma notícia difícil é importante que seja uma figura que acredite nas potencialidades desses pacientes, bem como no que de melhor possa fazer com tal informação. Um dos requisitos principais na comunicação é informar as questões consideradas pesarosas de forma clara e verdadeira, não omitindo prognósticos e percursos possíveis. Ao mesmo tempo, deve-se inspirar respeito por meio da exclusividade dispensada por aquele indivíduo, reconhecendo-o e respeitando-o como um ser único, tendo na prática o firmamento de que tudo foi feito para salvar sua vida. E, por fim, deixar-se proporcionar ao outro a generosidade, que se solidariza e identifica-se com a dor do outro.

É importante ressaltar que os problemas de comunicação apresentados pelos profissionais da saúde também são fruto de uma insuficiência na formação acadêmica, na qual é necessário oferecer maior ênfase no desenvolvimento destas habilida- des de comunicação, como também em uma orientação para o atendimento mais humanizado desses profissionais. Atualmente não é suficiente levar em conta somente a eficiência médica, mas também a forma com que esses profissionais estabelecem um vínculo com seus pacientes e familiares.

Com isto, fazendo as aproximações das três atitudes facilitadoras propostas por Rogers é possível que haja mais estudos referentes à implementação dessa abordagem nas práticas dos protocolos, contribuindo para uma melhor e mais empática formação desses profissionais. Desse modo, por mais desafiador que seja, cabe a eles mostrar um interesse maior pelo outro e aceitar estar ligado ao paciente como uma pessoa digna de atenção, consideração e respeito.

Recomenda-se que tais considerações endereçadas neste estudo teórico possam compor um repertório para a construção de propostas interventivas junto a equipes de profissionais de saúde, bem como delineamentos de disciplinas e treinamentos em serviço, o que deve ser avaliado e aperfeiçoado constantemente, também compondo evidências para a realização de estudos empíricos no futuro. A investigação acerca dessas propostas para a melhoria da comunicação de más notícias entre profissionais de saúde e pacientes/clientes/usuários também pode ser refletida tendo em mente o público atendido, bem como os familiares, em uma apreensão mais complexa e sistêmica acerca dos processos de comunicação e humanização em saúde.

\section{Referências}

Albert Einstein Instituto Israelita de Ensino e Pesquisa (s/d). Estratégias de planejamento. São Paulo: Autor. Manuscrito não-publicado.

Almeida, L. R. (2009). Consideração positiva incondicional no sistema teórico de Carl Rogers. Temas em Psicologia, 17(1), 177-190.

Andreoli, P. B. A., \& Erlichman, M. R. (2008). Comunicação entre paciente, família e equipe no CTI. In L. K. Kitajima \& M. Cosmo (Orgs.), Psicologia e humanização assistência aos pacientes graves (pp. 101112). São Paulo: Atheneu.

Azeredo, N. S. G., Rocha, C. F., \& Carvalho, P. R. A. (2011). O enfrentamento da morte e do morrer na formação de acadêmicos de medicina. Revista Brasileira de Educação Médica (Rio de Janeiro), 35(1), 37-43.

Baile, W. F., Buckman, R., Lenzi, R., Globera, G., Beale, E. A., \& Kudelka, A. P. (2000). SPIKES: A six-step protocol for delivering bad News - application to the patient with cancer. The Oncologist, 5(4),302-11

Bezerra, M. E. S., \& Bezerra, E. N. (2012). Aspectos humanistas, existenciais e fenomenológicos presentes na abordagem centrada na pessoa. Revista NUFEN, 4(2), 21-36.

Buckman, R. (1992). How to break bad news: a guide for health care professions. Baltimore: John Hopkins Press. 
Burlá, C. (2006). Comunicação ao fim da vida. Prática Hospitalar (São Paulo), 8(43), 110-113.

Borges, M. S., Freitas, G., \& Gurgel, W. (2012). A comunicação da má notícia na visão dos profissionais de saúde. Revista Tempus Actas de Saúde Coletiva, 6(3),113-126.

Carneiro, A. C. M. S. (2017). Comunicação de más notícias no serviço de urgência. Dissertação de mestrado, Instituto Politécnico de Viana do Castelo, Portugal.

Cavalcante, M., Vasconcelos, M. V. L, \& Grosseman, S. (2017). A comunicação de más notícias por estudantes de Medicina: um estudo de caso. Dissertação de mestrado, Ensino na saúde da Faculdade de Medicina da Universidade Federal de Alagoas, Maceió.

Cruz, C. O., \& Riera, R. (2016). Comunicando más notícias: o protocolo SPIKES. Diagnóstico \& Tratamento, 21(3), 106-108.

Espinha, T. G. (2007). Vivências de internação de adultos em hospital geral: repensando o cuidado. Dissertação de mestrado, Centro de Ciências da Vida, Pontifícia Universidade Católica, Campinas, SP.

Fontgalland, R. C., \& Moreira, V. (2012). Da empatia à compreensão empática: evolução do conceito no pensamento de Carl Rogers. Memorandum (Belo Horizonte), (23), 32-56.

Kübler-Ross, E. (2008). Sobre a morte e o morrer (3ª ed.). São Paulo: Martins Fontes.

Leal-Seabra, F., \& Costa, M. J. (2015). Comunicação de más notícias pelos médicos no primeiro ano de internato: um estudo exploratório. Revista de la Fundación Educación Médica, 18(6), 387-395.

Lech, S. S., Destefani, M. S., \& Bonamigo, E. L. (2013). Percepção dos médicos sobre comunicação de más notícias ao paciente. Unoesc \& Ciência - ACBS, 4(1), 69-78.

Lino, C. A., Augusto, K. L., Oliveira, R. A. S., Feitosa, L. B., \& Caprara, A. (2010). Uso do protocolo SPIKES no ensino de habilidades em transmissão de más notícias. Revista Brasileira de Educação Médica, 35(1), 52-57.

Mendes, K. D. S., Silveira, R. C. C. P., \& Galvão, C. M. (2008). Revisão integrativa: método de pesquisa para a incorporação de evidências na saúde e na enfermagem. Texto \& Contexto Enfermagem, 17(4), 758-764.

Mochel, E. G., Perdigão, E. L. L., Cavalcanti, M. B., \& Gurgel, W. B. (2010). Os profissionais de saúde e a má notícia: estudo sobre a percepção da má notícia na ótica dos profissionais de saúde em São Luís/MA. Cadernos de Pesquisa, 17(3), 47-56.

Monteiro, D. T., \& Quintana, A. M. (2016). A comunicação de más notícias na UTI: Perspectiva dos médicos. Psicologia: Teoria e Pesquisa, 32(4), 1-9.
Moreira, V. (2010). Revisitando as fases da abordagem centrada na pessoa. Estudos de Psicologia (Campinas), 27(4), 537-544.

Pereira, A. T. G., Fortes, I. F. L., \& Mendes, J. M. G. (2013). Comunicação de más notícias: Revisão sistemática da literatura. Revista de Enfermagem UFPE, 7(1), 227-35.

Pereira, C. R. (2010). Comunicando más notícias: protocolo PACIENTE. Tese de doutorado, Faculdade de Medicina de Botucatu, Universidade Estadual Paulista, Botucatu, SP.

Pereira, M. A. G. (2005). Má noticia em saúde: um olhar sobre as representações dos profissionais de saúde e cidadãos. Texto $\&$ Contexto Enfermagem, 14(1), 3337.

Petrilli, A. S., Pascalicchio, A. P. A., Dias, C. G., \& Petrilli, R. T. (2000). O processo de comunicar e receber o diagnóstico de uma doença grave. Diagnóstico $\mathcal{E}$ Tratamento, 5(1), 35-39.

Pinheiro, U. M. S. (2012). Más notícias em oncologia: o caminho da comunicação na perspectiva de médicos e enfermeiros. Dissertação de mestrado, Cuidado, Educação em Enfermagem e Saúde, Universidade Federal de Santa Maria, Santa Maria, RS.

Ramos, A. P., \& Bortagarai, F. M. (2012). A comunicação não-verbal na área da saúde. Revista CEFAC, 14(1),164-170.

Rogers, C. R. (1973). Tornar-se pessoa. São Paulo: Martins Fontes.

Rogers, C. R. (1977). Uma maneira negligenciada de ser: a maneira empática. In C. R. Rogers, \& R. L. Rosenberg, A pessoa como centro (pp. 69-89). São Paulo: EPU.

Rogers, C. R. (2012). Um jeito de ser. São Paulo: E.P.U. (Original publicado em 1980).

Rogers, C. R., \& Stevens, R. B. (1978). De pessoa para pessoa São Paulo: Pioneira.

Rodriguez, M. I. F. (2014). Despedida silenciada: Equipe médica, família, paciente - cúmplices da conspiração do silêncio. Psicologia Revista - Revista da Faculdade de Ciências Humanas e da Saúde, 23(2), 261-272.

Scorsolini-Comin, F. (2014). Guia de orientação para iniciação científica. São Paulo: Atlas.

Scorsolini-Comin, F. (2015). Aconselhamento psicológico: aplicações em gestão de carreiras, educação e saúde. São Paulo: Atlas.

Silva, M. J. P. (2002). A comunicação na área da saúde. In L. R. Bebb (Org.), Comunicação tem remédio (pp. 13-19). São Paulo: Gente.

Silva, L. M. G., Brasil, V. V., Guimarães, H. C. Q. C. P., Savonitti, B. H. R. A., \& Silva, M. J. P. (2000). Comunicação não-verbal: reflexões acerca da linguagem corporal. Revista Latino-americana de Enfermagem (Ribeirão Preto), 8(4), 52-58. 
Sombra Neto, L. L., Silva, V. L. L., Lima, C. D. C., Moura, H. T. M., Gonçalves, A. L. M., Pires, A. P. B., Fernandes, V. G. (2017). Habilidade de comunicação da má notícia: o estudante de Medicina está preparado? Revista Brasileira de Educação Médica, 41(2), 260-268.

Souza, L. V., \& Santos, M. A. (2013). Quem é o especialista? Lugares ocupados por profissionais e pacientes no tratamento dos transtornos alimentares. Estudos de Psicologia, $18(2), 259-267$

Souza, L. V., \& Scorsolini-Comin, F. (2011). Relações profissionais em equipes de saúde: alternativas construcionistas relacionais. Saúde \& Transformação Social / Health \& Social Change, 1(3), 37-46.

Stefanelli, M., \& Carvalho, E. (2012). A comunicação nos diferentes contextos da enfermagem. São Paulo: Manole.

Trzan-Ávila, A., \& Jacó-Vilela, A. M. (2012). Uma história da Abordagem Centrada na Pessoa no Brasil. Estudos e Pesquisas em Psicologia, 12(3), 1063-1069.

Victorino, A., Nisenbaum E., Gibello J., Bastos M., \& Andreoli P. (2007). Como comunicar más notícias: revisão bibliográfica. Revista da Sociedade Brasileira de Psicologia Hospitalar, 10(1), 53-63.
Vanessa Jaqueline Calsavara é Psicóloga pela Universidade Federal do Triângulo Mineiro. E-mail: vanessajaqueline.c@hotmail.com.

Fabio Scorsolini-Comin é Docente do Departamento de Enfermagem Psiquiátrica e Ciências Humanas e do Programa de Pós-graduação em Enfermagem Psiquiátrica da Escola de Enfermagem de Ribeirão Preto da Universidade de São Paulo. Doutor em Psicologia pela Universidade de São Paulo. E-mail: fabio.scorsolini@usp.br

Carlos Alexandre Curylofo Corsi é Mestre pelo Programa de Pós-graduação em Enfermagem Psiquiátrica da Escola de Enfermagem de Ribeirão Preto da Universidade de São Paulo. Técnico em Banco de Tecidos Humanos no Banco de Tecidos do Hospital das Clínicas da Faculdade de Medicina de Ribeirão Preto (HCFMRP-USP). E-mail: carlos_ccorsi@hotmail.com.

Recebido em 09.02.2018

Primeira Decisão Editorial em 04.04.2018

Aceito em 04.05.2018 